\title{
Accuracy and Efficacy of Software-guided Bony Realignment in Periarticular Deformities of the Lower Limb
}

\author{
Pritish Singh ${ }^{1}$, Dhananjay Sabat ${ }^{2}$, Saurabh Dutt ${ }^{3}$, Rakesh Sehrawat ${ }^{4}$, Balu Prashanth ${ }^{5}$, Anubhav Vichitra ${ }^{6}$, Vinod Kumar ${ }^{7}$
}

\begin{abstract}
Aim and objective: Software-guided realignment is proposed as an easy and accurate method of achieving simultaneous multiaxial correction. The accuracy and efficacy in periarticular problems have not been investigated fully. This study investigates the results and possible clinical benefits. Materials and methods: A retrospective review was performed in 24 patients with 27 periarticular deformities of the lower limb treated by the llizarov technique. Bony realignment was achieved by a software-guided hexapod realignment device. The deformity category, deformity severity score (DSS) and individual deformity component scores were measured for objective quantification of each deformity. The periarticular level, number of manoeuvres, correction period and any difficulties in the execution of the correction manoeuvre were noted. Pre-procedure and post-procedure values of deformity parameters were analysed to estimate the accuracy and efficacy of the realignment device.

Results: The correction manoeuvre was accomplished successfully in all patients except two. The mean correction period was 14.9 days (range, 5-38 days). The mean pre-procedure DSS was 18.7 (range 6.3-27.3), which reduced to a mean post-procedure value of 1.5 (range, $0-7.9$ ) with a $92.0 \%$ deformity correction ( $p$-value $<0.001)$ ). There was a significant reversal of individual deformity components. DSS values were achieved to an excellent level $(<3.5)$ in 25 deformities and to good and poor levels in one deformity, respectively.

Conclusion: Software-guided realignments are effective for accurate realignment of periarticular deformities using llizarov fixators. These devices offer simultaneous multidimensional corrections even in complex multiplanar deformities and simplify the task of deformity correction. Clinical significance: The present study assesses the accuracy and efficacy of software-guided realignments using novel concepts of deformity category, DSS and individual deformity component scores, which may overcome some of the shortcomings of conventional assessment methods. Keywords: Deformity severity score, llizarov fixator, Ortho-SUV frame, Periarticular deformities, Software-guided bony realignments. Strategies in Trauma and Limb Reconstruction (2021): 10.5005/jp-journals-10080-1524
\end{abstract}

\section{INTRODUCTION}

Bony malalignment can result from periarticular open fractures, nonunions and physeal arrests. ${ }^{1}$ Such malalignment has a direct impact on the outcome and warrants consideration for correction. ${ }^{2}$ Concomitant infection, compromised soft tissue cover, compromised vascularity, disturbed metabolic milieu and systemic host factors add to the complexity of the treatment. Bony deformities in these conditions are usually multiplanar and complex to realign and often require the use of Ilizarov techniques. ${ }^{1,3}$

Conventional correction of such multiplanar deformities requires custom-configured llizarov frame constructs for each component of the deformity, with repeated frame transformations cumbersome and time-consuming. In some cases, the complexity of frame construction may necessitate the acceptance of suboptimal alignment, orientation or bone contact. ${ }^{4,5}$

Software-guided realignment devices, like the Taylor spatial frame (TSF), the Ortho-SUV frame, etc., solve the problem of repeated frame transformation and offer a simultaneous correction of all deformity components even in complex scenarios. ${ }^{1-6}$ The present study reviews the accuracy and efficacy of using a softwareguided realignment device in complex periarticular deformities of the lower limb and the possible benefits offered to the patient by such technology.

\section{Materials and Methods}

Periarticular deformities of lower limb long bones treated using an Ilizarov ring fixator from October 2016 to February 2020 time period were retrospectively reviewed. Cases of deformity with
${ }^{1-7}$ Department of Orthopaedic Surgery, Maulana Azad Medical College and Associated Lok Nayak Hospital, New Delhi, India

Corresponding Author: Pritish Singh, Department of Orthopaedic Surgery, Maulana Azad Medical College and Associated Lok Nayak Hospital, New Delhi, India, Phone: +91 8010437241, e-mail: mamc5279@gmail.com

How to cite this article: Singh P, Sabat D, Dutt S, et al. Accuracy and Efficacy of Software-guided Bony Realignment in Periarticular Deformities of the Lower Limb. Strategies Trauma Limb Reconstr 2021;16(2):65-70.

Source of support: Nil

Conflict of interest: None

periarticular involvement, for example, open periarticular fractures, nonunion, malunion, post-traumatic physeal arrests and metabolic disorder-related angular deformities, were included. Cases treated by conventional correction methods and those cases in which an acute intra-operative correction was achievable were excluded. Only cases in which a software-guided realignment device was used for gradual deformity correction were assessed. Written informed consent was obtained from all the patients. The Institutional Ethical Board approved the study.

Twenty-four patients with 27 periarticular lower limb deformities were treated with a software-guided realignment device. There was no loss to follow-up. The Ortho-SUV frame (Ortho-SUV Ltd. \& Vreden Russian Research Institute of Traumatology and Orthopaedics, St. Petersburg, Russian Federation) was used in all patients. ${ }^{7}$ 
Each deformity was assessed based on the presence of deformity components of translation, angulation, rotation and axial length deformity. Translation, angulation and axial length mismatch (either overriding or distraction) were measured using orthogonal X-rays, whilst rotational malalignment was evaluated using clinical methods. The overall limb length discrepancy was excluded from the analysis.

\section{Concept of a Deformity Severity Score}

Objective quantification of the deformity was done with respect to a reference deformity. The reference deformity was conceptualised as having translation equal to the diameter of the major bone fragment in both orthogonal planes, angulation of $45^{\circ}$ in both orthogonal planes, a rotation deformity of $45^{\circ}$ and axial length mismatch (either distraction or overriding) equal to the major fragment diameter in both orthogonal planes. This reference deformity was assigned a severity score of 100 points.

Each deformity was quantified with respect to this reference deformity. The component score was calculated for all four components of deformity by taking the mean of their percentage values in the orthogonal planes. The mean value of all four component scores for a deformity constituted the deformity severity score (DSS). A higher score depicted a more severe deformity and a lesser score the converse. An illustrative example of DSS calculation is given in Table 1 using pre-procedure radiographs (Figs $1 \mathrm{~A}$ and $1 \mathrm{~B}$ ) and post-procedure radiographs (Figs 1C and 1D) in orthogonal planes.

\section{Technical Tips for Deformity Severity Score Calculation}

Orthogonal X-rays of the limb segment with optimal visualisation of both bony fragments are needed for deformity quantification with respect to the DSS. The major fragment along with its anatomical axis and transverse diameter at corticotomy or fracture end is identified in orthogonal planes. Translation, angulation and axial length deformity (overlapping or distraction) are measured in both planes individually along with total a rotational component
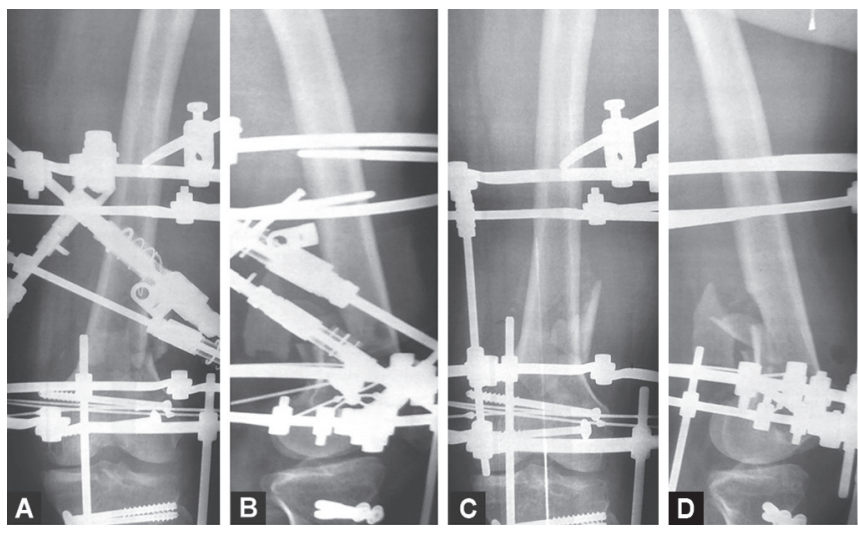

Figs $1 A$ to $D$ : (A and B) Pre-procedure anteroposterior view $(A)$ and lateral view (B) radiograph of a patient with distal femur periarticular deformity for DSS calculation. Major fragment diameter (distal fragment) was $46 \mathrm{~mm}$, and it has translation of $2 \mathrm{~mm}$ and $18 \mathrm{~mm}$ in AP and lateral views, respectively. Anatomic axis malangulation was estimated to be $3^{\circ}$ and $4^{\circ}$ in AP and lateral views, respectively. There was axial length mismatch (overriding) of $6 \mathrm{~mm}$ and $8 \mathrm{~mm}$ in AP view and lateral views, respectively. Rotational malalignment was evaluated clinically and found to be $5^{\circ}$. Individual component scores were calculated. All four components of deformity were present, and it was characterised as type $4 C$ deformity with DSS 23.8; (C and D) Post-procedure radiographs in anteroposterior (C) and lateral (D) views after achieving bony realignment using software-guided realignment device for DSS calculation. Major fragment diameter (distal fragment) was $38 \mathrm{~mm}$, and it has residual translation of $1 \mathrm{~mm}$ and $2 \mathrm{~mm}$ in AP and lateral views, respectively. Anatomic axis malangulation was estimated to be $0^{\circ}$ and $2^{\circ}$ in AP and lateral views, respectively. There was axial length mismatch (overriding) of $0 \mathrm{~mm}$ and $3 \mathrm{~mm}$ in AP view and lateral views, respectively. Rotational malalignment was found to be completely reversed to normal values. Individual component scores were recalculated; two components (angulation and rotation) have reversed below significant level, whilst translation and axial length deformity also reversed till residual component scores of $3.7(92.9 \%$ reversal) and 3.9 (77.1\% reversal), respectively. The post-procedure residual deformity was type 2 C with a DSS value of 2.4 with $89.9 \%$ DSS reversal

Table 1: DSS and individual deformity component score calculation using illustrative pre-procedure radiographs (Figs $1 \mathrm{~A}$ and $1 \mathrm{~B}$ ) and postprocedure radiographs (Figs $1 \mathrm{C}$ and 1D)

\begin{tabular}{|c|c|c|c|c|c|c|c|c|c|c|c|}
\hline \multirow[b]{3}{*}{ Component } & \multicolumn{11}{|c|}{$\begin{array}{l}\text { Major fragment diameter AP plane }{ }^{a}=46 \mathrm{~mm} \\
\text { Major fragment diameter Lat plane } e^{b}=38 \mathrm{~mm}\end{array}$} \\
\hline & \multicolumn{5}{|c|}{ Pre-procedure } & \multicolumn{6}{|c|}{ Post-procedure } \\
\hline & & $\begin{array}{l}\text { Component } \\
\text { value }\end{array}$ & $\begin{array}{c}\text { Deformity } \\
\text { percentage in } \\
\text { orthogonal } \\
\text { planes }\end{array}$ & $\begin{array}{l}\text { Component } \\
\text { score }\end{array}$ & $\begin{array}{c}\text { Deformity } \\
\text { severity } \\
\text { score }\end{array}$ & & $\begin{array}{c}\text { Component } \\
\text { value }\end{array}$ & $\begin{array}{c}\text { Deformity } \\
\text { percentage in } \\
\text { orthogonal } \\
\text { planes }\end{array}$ & $\begin{array}{l}\text { Component } \\
\text { score }\end{array}$ & $\begin{array}{l}\text { Reversal } \\
\text { percent- } \\
\text { ages (\%) }\end{array}$ & $\begin{array}{c}\text { Deformity } \\
\text { severity score/ } \\
\text { deformity } \\
\text { category }\end{array}$ \\
\hline \multirow[t]{2}{*}{ Translation } & $\mathrm{AP}$ & $2 \mathrm{~mm}$ & 4.3 & 51.6 & & $\mathrm{AP}$ & 1 & 2.2 & 3.7 & 92.9 & \\
\hline & Lat & $18 \mathrm{~mm}$ & 47.3 & & & Lat & 2 & 5.3 & & & \\
\hline \multirow[t]{2}{*}{ Angulation } & AP & $3^{\circ}$ & 6.7 & 15.6 & & $\mathrm{AP}$ & 0 & 0 & 2.2 & 85.9 & 2.4 \\
\hline & Lat & $4^{\circ}$ & 8.9 & & 23.8 & Lat & 2 & 4.4 & & & Type 2C \\
\hline \multirow{2}{*}{$\begin{array}{l}\text { Axial } \\
\text { length } \\
\text { deformity }\end{array}$} & AP & $6 \mathrm{~mm}$ & 13.0 & 17.0 & Type 4C & AP & 0 & 0 & 3.9 & 77.1 & $89.9 \%$ \\
\hline & Lat & $8 \mathrm{~mm}$ & 21.1 & & & Lat & 3 & 7.9 & & & reversal \\
\hline Rotation & & $5^{\circ}$ & 11.1 & 11.1 & & & 0 & 0 & 0 & 100 & \\
\hline
\end{tabular}

${ }^{\mathrm{a} A P}$, anteroposterior plane

bat, lateral plane 
deformity. The deformity percentage of each component is calculated separately with respect to the component value of the reference deformity. [For example, the percentage deformity for a translation of $5 \mathrm{~mm}$ in a bone with $20 \mathrm{~mm}$ diameter will be $25 \%$. Similarly, a $22.5^{\circ}$ angulation (half of reference deformity angulation) will constitute an angulation deformity percentage of 50\%]. The mean value of the orthogonal deformity percentages for each component will constitute the component score. A higher score correlates with severe deformity, and a lower score correlates with a smaller deformity with respect to that component. A mean of all four individual component scores will produce a composite score for the deformity viz., the DSS. A higher DSS correlates with severe deformity, and a smaller score will correlate with a lesser deformity. Individual component scores and DSS values convey the relative proportion of each component in a deformity and guide for characterisation of deformity in five grades.

\section{Deformity Characterisation}

An individual deformity component score $\geq 3.5$ was considered significant and established as a marker of a clinically significant deformity. From the deformity components (translation, angulation, rotation and axial length deformity), we divided each deformity into five categories by increasing the complexity, that is, no deformity (0C), one-component deformity (1C), two-component deformity (2C), three-component deformity (3C) and four component deformity (4C).

\section{Correction Manoeuvre and its Assessment}

The end point of the correction was based on the goals of treatment as used by Paley et al. for lower limb deformities. ${ }^{8}$ The goal was to realign the nearby joint orientation to within $3^{\circ}$ of normal values. The end point was individualised for each case taking into account influences like the concomitant need of optimal bony contact and soft tissue concerns.

The Ortho-SUV correction device was applied in the postoperative period as a bedside procedure. Threaded rods at deformity level were taken out after application of Ortho-SUV struts, and specific X-rays, as recommended in the software, were taken. The correction through the Ortho-SUV device was planned and executed as per the software prescription. Repeat software planning (residual mode planning) was done till the goal of correction could be achieved.

The number of manoeuvres, the correction period and difficulties in the execution of the correction were noted. A deformity that did not respond to correction after three attempts was considered as an indication to abandon the software-guided manoeuvre. After achieving the goal of bony realignment, OrthoSUV struts were replaced with threaded connecting rods, and post-procedure $\mathrm{X}$-rays were taken.

The pre-procedure and post-procedure values of deformity parameters were measured and analysed. The accuracy of the software-guided realignment device was obtained by calculating changes in component scores and DSS.

Efficacy was calculated as the capability of hardware to bring the deformity to the correction goal level. As such, the efficacy of correction was categorised on the basis of the reversal percentage of the residual DSS: This was excellent (post-procedure DSS $<3.5$ with component reversal $>80 \%$ for $1 \mathrm{C}$ and $2 \mathrm{C}$ grades OR DSS reversal $>80 \%$ (for $3 C$ and $4 C$ grades); good (post-procedure DSS
$>3.5$ with reversal $>80 \%$ ), fair (post-procedure DSS $>3.5$ with reversal $71-80 \%$ ) and poor (post-procedure DSS $>3.5$ with reversal $\leq 70 \%$ ).

\section{Statistical Analysis}

Data analysis was performed using the SPSS version 20.0. Descriptive statistics used mean (range: min-max) and number (percentage). Continuous variables were analysed using the Student's $t$-test. A $p$ value of less than 0.05 was considered statistically significant.

\section{Results}

Twenty-seven periarticular deformities in twenty-four patients (male, sixteen; female, seven; and transgender, one) with a mean age of 29.8 years (range, 12-55 years) were treated. The deformity levels were the proximal femur (3.7\%), distal femur (25.9\%), proximal tibia (37.0\%) and distal tibia (33.3\%). Clinical aetiologies comprised open fracture (18.5\%), malunited fractures $(11.1 \%)$, infected nonunion (18.5\%), aseptic nonunion (18.5\%), metabolic disorder-related angular deformities (18.5\%) and physeal growth arrest-related deformities (14.8\%). The initial demographic details and clinical characteristics of the patients are depicted in Table 2.

The distribution of deformity grading was $1 \mathrm{C}(3.7 \%), 2 \mathrm{C}(14.8 \%)$, 3C (40.7\%) and 4C (40.7\%). The mean pre-procedure DSS was 18.7 (range, 6.3-27.3). The mean translation component score was 18.8 (range, 0-43.7); the mean angulation score, 24.7 (range, 8.9-40); the mean axial length deformity score, 22.1 (range, 0-50.0); and the mean rotational deformity score, 8.5 ( range, $0-22.2$ ).

A correction was accomplished in all patients except two patients. One patient with a proximal femoral deformity required hardware removal before completion of correction due to abutment on the medial side by protruding fixator struts (Fig. 2). In the other patient, strut length exhaustion was noted to be the reason. None of the patients had hardware-related issues like stability compromise or breakage requiring abandonment of the procedure (Fig. 3).

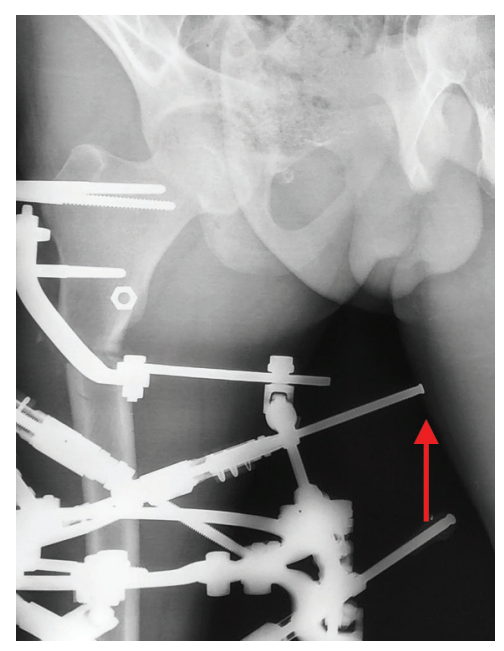

Fig. 2: External rotation radiograph of a patient with proximal femur periarticular deformity showing strut of software-guided realignment device impinging on the thigh (arrow), which required apparatus removal before completion of correction manoeuvre 
Table 2: Initial patient demographics and procedure characteristics

\begin{tabular}{|c|c|}
\hline Parameter & Observation/value \\
\hline No of patients & 24 \\
\hline Deformities & 27 \\
\hline Age & 29.8 years (range, $12-55$ years) \\
\hline $\begin{array}{l}\text { Gender } \\
\text { Male } \\
\text { Female } \\
\text { Transgender }\end{array}$ & $\begin{array}{c}66.7 \%(n=16) \\
29.2 \%(n=7) \\
4.1 \%(n=1)\end{array}$ \\
\hline $\begin{array}{l}\text { Clinical entities } \\
\text { Fractures } \\
\text { Infected nonunion } \\
\text { Aseptic nonunion } \\
\text { Malunion } \\
\text { Physeal arrest } \\
\text { Metabolic disease-related angular } \\
\text { limb deformities }\end{array}$ & $\begin{array}{l}18.5 \%(n=5) \\
18.5 \%(n=5) \\
18.5 \%(n=5) \\
11.1 \%(n=3) \\
14.8 \%(n=4) \\
18.5 \%(n=5)\end{array}$ \\
\hline $\begin{array}{l}\text { Deformity grading } \\
1 C \\
2 C \\
3 C \\
4 C\end{array}$ & $\begin{array}{l}3.7 \%(n=1) \\
14.85(n=4) \\
40.7 \%(n=11) \\
40.7 \%(n=11)\end{array}$ \\
\hline $\begin{array}{l}\text { Periarticular level } \\
\text { Proximal femur } \\
\text { Distal femur } \\
\text { Proximal tibia } \\
\text { Distal tibia }\end{array}$ & $\begin{array}{l}3.7 \%(n=1) \\
25.9 \%(n=7) \\
37.0 \%(n=10) \\
33.3 \%(n=9)\end{array}$ \\
\hline $\begin{array}{l}\text { Fracture/corticotomy } \\
\text { Fracture } \\
\text { Corticotomy }\end{array}$ & $\begin{array}{l}55.5 \%(n=15) \\
44.4 \%(n=12)\end{array}$ \\
\hline $\begin{array}{l}\text { No of manoeuvres needed } \\
\text { One manoeuvre } \\
\text { Two manoeuvres } \\
\text { Three manoeuvres }\end{array}$ & $\begin{array}{c}55.5 \%(n=15) \\
37.0 \%(n=10) \\
7.4 \%(n=2)\end{array}$ \\
\hline $\begin{array}{l}\text { Manoeuvre complications } \\
\text { Incomplete procedure } \\
\text { Hardware failure/breakage }\end{array}$ & $\begin{array}{l}7.4 \%(n=2) \\
0\end{array}$ \\
\hline $\begin{array}{l}\text { Efficacy } \\
\text { Excellent } \\
\text { Good } \\
\text { Fair } \\
\text { Poor }\end{array}$ & $\begin{array}{l}92.6 \%(n=25) \\
3.7 \%(n=1) \\
0 \\
3.7 \%(n=1)\end{array}$ \\
\hline Overall correction period (in days) & 14.9 (range, 5-38), SD: 7.4 \\
\hline $\begin{array}{l}\text { Correction period deformity } \\
\text { gradewise (in days) } \\
1 C \\
2 C \\
3 C \\
4 C\end{array}$ & $\begin{array}{l}18 \\
15.2 \text { (range, 11-22), SD: } 5.4 \\
15.8 \text { (range, 7-38), SD: } 8.9 \\
13.5 \text { (range, 5-26), SD: } 7.1\end{array}$ \\
\hline $\begin{array}{l}\text { Correction period (case wise) } \\
\text { (in days) } \\
\text { Fracture-related cases } \\
\text { Corticotomy-related cases }\end{array}$ & $\begin{array}{l}10.5 \text { (range, 5-18), SD: } 3.9 \\
20.4 \text { (range, 10-38), SD: } 7.2\end{array}$ \\
\hline
\end{tabular}

The deformity was corrected in a single prescription from the software in $58.3 \%$ of cases $(n=15)$; one additional software planning procedure (residual deformity mode of software) was needed in $41.7 \%$ of cases $(n=10)$ and a third in two patients.

The mean correction period was 14.9 days (range, 5-38 days) for all deformities. The correction period for fracture-related cases

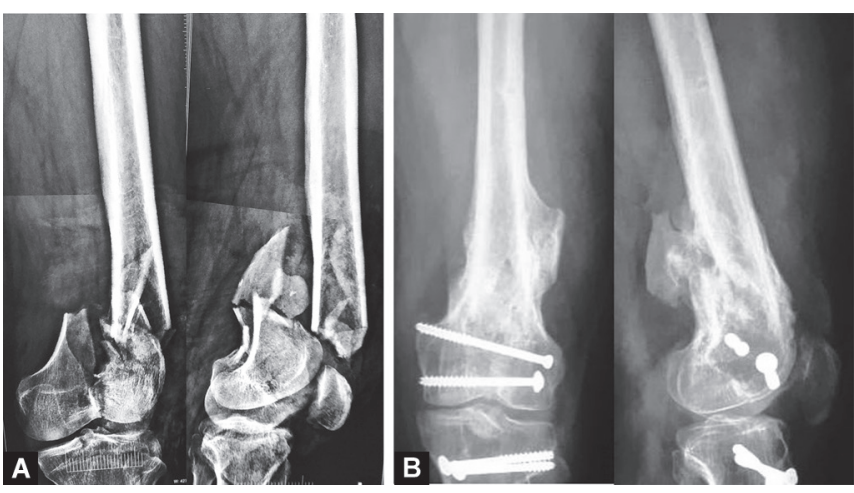

Figs $3 \mathrm{~A}$ and $\mathrm{B}$ : Injury radiograph (A) and 1-year follow-up radiograph (B) of the same patient with open periarticular fracture treated using Ilizarov fixator and software-guided realignment

(mean, 10.5 days; range, 5-18 days) was significantly less than corticotomy-related cases( mean, 20.4 days; range, 10-38 days).

The joint orientation angle with respect to the anatomical axis of the bone was measured for uniformity. This was successfully reversed to $\leq 3^{\circ}$ of normal in $92.6 \%$ of cases $(n=25)$. The mean post-procedure DSS was 1.5 (range, $0-7.9$ ) with a $92.0 \%$ reversal ( $p$-value $<0.005)$. There was a significant reversal of individual deformity components (Table 3). The efficacy of software-guided realignment reached an excellent level $(<3.5)$ in 25 deformities, a good level in 1 patient (DSS value of 3.8) and poor in one patient (DSS value of 7.9).

\section{Discussion}

This study indicates that software-guided devices like the OrthoSUV to be accurate and effective for achieving bony realignment in the periarticular deformities of the lower limb. The spectrum of deformities varied in severity and complexity, but all components, namely translation, angulation, malrotation and axial length mismatch (distracted or overlapping fragments), were optimally reversed using the software-guided realignment method.

The procedure was able to address simultaneously all components of deformity for all the cases. Translation, angulation and rotation were corrected to the end point level in the majority of cases. Axial length mismatch was the most common deformity requiring a repeat run of the software programme. This under correction of axial length mismatch with the first prescription generated by the software was considered as a protective mechanism against bone collision or under correction of the other deformity components. Any residual deformity was corrected easily with the same hardware assembly.

Simultaneous correction of all components of a deformity with software-guided realignment is a major advantage over conventional correction methods. Conventional methods have difficulty in terms of planning and their execution with an estimation of the accurate plane of deformity, accurate hinge placement and repeated frame transformation being some of the difficulties faced with. ${ }^{4-7}$

Software-guided realignment devices reliably overcome the difficulties of conventional methods. Some error-prone tasks are directed to the software to resolve in realignment devices like Ortho-SUV, TSF, etc. Software-based corrections do not require manual identification of deformity plane and position of the centre of rotation of angulation, with some suggestion that this 
Table 3: Pre-procedure and post-procedure deformity characteristics

\begin{tabular}{|c|c|c|c|c|c|c|c|}
\hline & \multicolumn{3}{|c|}{ Pre-procedure } & \multicolumn{3}{|c|}{ Post-procedure } & \multirow[b]{2}{*}{$p$-value } \\
\hline & Mean & Range & $S D$ & Mean & Range & $S D$ & \\
\hline Deformity severity score & 18.7 & $6.3-27.3$ & 8.7 & 1.5 & $0-7.9$ & 1.6 & $<0.001$ \\
\hline \multicolumn{8}{|l|}{ Component scores } \\
\hline Translation score & 18.8 & $0-43.7$ & 14.2 & 1.6 & $0-5.4$ & 2.1 & $<0.001$ \\
\hline Angulation score & 24.7 & $8.9-40.0$ & 14.3 & 2.1 & $0-6.8$ & 2.1 & $<0.001$ \\
\hline Axial length deformity score & 22.1 & $0-50.0$ & 16.5 & 2.4 & $0-25$ & 5.1 & $<0.001$ \\
\hline Rotation score & 8.5 & $0-22.2$ & 11.1 & 0 & - & 0 & - \\
\hline
\end{tabular}

may reduce radiographic exposures needed for the evaluation of deformity of the patient. Orthogonal radiographs of deformity are required and used as primary data sources by software for correction planning. Deformity corrected in orthogonal planes gets corrected in its true oblique plane also.,8

Software-guided realignment procedures could accomplish both acute and gradual corrections well without any hardware issues. Acute corrections were planned for clinical problems of lesser magnitude with conducive soft tissue cover or those associated with fracture or osteotomy, whilst gradual corrections were planned for conditions associated with corticotomy.

We have used the deformity component score and DSS for objective quantification of deformity and outcome analysis. Conventionally, the quantification of deformity is done in terms of joint line orientations, anatomical axis and mechanical axis deviations as described by Paley. Another method is an individual assessment of each deformity component. ${ }^{9,10}$ The authors feel that individual bone dimensions need consideration in the quantification of each deformity in order for uniform comparative evaluation. This overcomes the quandary of a similar component value effecting a different deformity severity for two different diameter bones: For example, a translation of $5 \mathrm{~mm}$ will have different deformity severity in bone areas like the diaphyseal femur and metaphyseal femur or in the same bone of differently sized individuals.

Paley assessed the accuracy of corrections in lower limb deformities treated by llizarov methods and used parameters of mechanical axis deviation, deviation in joint line orientation and tibiofemoral angle for outcome analysis. ${ }^{9}$ We found these parameters nonapplicable on the majority of our patients owing to the frequent occurrence of flexion deformity, fixed contractures and the patient's inability to undertake full extension weight-bearing $\mathrm{X}$-rays of the lower limb.

The majority of periarticular conditions treated by the llizarov method are likely to have some degree of joint contractures with the exception of a fresh fracture. Due to these practical shortcomings, treatment planning with respect to the anatomical axis is more pragmatic. Parameters used by Paley for accurate assessments like mechanical axis deviation and tibiofemoral angle cannot be uniformly applied for outcome analysis in periarticular deformities. Mechanical axis considerations may be applied only when the clinical scenario allows its unhindered estimation.

In light of these shortcomings and practical concerns, we have devised and used a deformity component score and DSS for objective quantification of deformity and its outcome. In this system, we have conceptualised a reference deformity that has all components of a deformity with a 100-point score.

The limits of reference deformity were set arbitrarily through considering the likely range of severity of the majority of deformities in clinical practice. It incorporates native bone dimensions in the severity estimation and obviates the need for full extension, weight-bearing X-rays. Deformity quantification in nonfracture cases can be done at the level of osteotomy and will correspond with displacements needed in correction manoeuvre. Translation, angulation and distraction or overriding can be measured easily with objective methods. The rotational component is measured by clinical methods rather than computerised tomography scanbased assessments. Pre-procedure and post-procedure scores allow a reliable assessment of the performance of realignment devices with respect to individual deformity components and to the overall deformity as well. Since DSS estimation obviates the needs of full-length weight-bearing radiographs and needs only deformity level estimations, its best suited for efficacy assessment of software-guided realignment devices.

Software-guided realignment devices have evolved over the last three decades. Initial prototypes of hexapod-type fixators like Phillipe Muniot device (1985) and Pisler-Kostin device (1989) were software independent and had limited clinical use. The TSF (1994) was the first software-guided realignment device and was used widely. Other devices like the Klaus Seide hexapod (1996), Eisenberg fixator (1998), Adam's Frame (2009), etc. did not gain widespread acceptance for clinical use. ${ }^{11,12}$

The Ortho-SUV frame is a newer-generation version of software-guided external fixator and is gaining widespread acceptance for clinical use due to its various unique features. It is modular, independent of the size and shape of rings and offers simultaneous multidimensional deformity correction even in complex deformities. The Ortho-SUV frame does not require prefabricated frame-specific rings for its application. Unlike the TSF, with the Ortho-SUV frame, specific X-rays need not be exactly parallel to the plane of rings or in truly orthogonal planes. It accepts some degree of variation in orthogonal radiographs. ${ }^{7}$ Ease in taking optimal X-ray for the Ortho-SUV frame decreases the X-ray exposure as compared to the TSF.

This device allows for fine adjustments up to a fraction of a millimetre in any plane including rotational movement. It provides optimisation of bone contact, axis shift and rotation adjustment along with pictorial visualisation of the bone trajectory during correction. ${ }^{7}$ In our opinion, the optimal time to apply a softwareguided hexapod is the post-operative period. This saves cost and avoids the need for the intra-operative application of the device for the surgeon. The Ortho-SUV strut application can be done as a bedside procedure and does not require anaesthesia.

We have found deformities of the proximal metaphyseal femur to be a poor candidate for applying the Ortho-SUV frame due to hardware-related issues. There was reliable and anticipated correction movement of bone in all of our patients 
with software-prescribed correction. The clinical possibility of suboptimal correction may be due to compromised stability of fixation or subtle errors of hardware measurements when entering data into the software.

\section{LIMITATIONS}

The main weaknesses of this study are the small sample size and heterogeneous group of deformities. Simultaneous assessment of all deformity grades may not reveal discreet outcomes amongst different severity groups. Axial length deformity may be divided into two subsets, that is, distraction and overriding for more detailed outcome analysis.

\section{Conclusion}

Software-guided corrections of malalignment are clinically reliable, accurate and effective for periarticular deformities treated using Ilizarov fixators. This method works reliably even in complex multiplanar deformities, offering simultaneous multidimensional corrections, and works well for acute as well as gradual corrections. The procedure helps to avoid the intricacies of conventional methods and simplifies the task of deformity correction.

\section{ACKnOWLedgement}

The authors would like to thank Dr. Manish Taywade for professional assistance in statistics calculations.

\section{Ethical Approval}

The study was reviewed and approved by Institutional Ethical Committee (IECID-F.1/IEC/MAMC/76/04/2020/No129; dated 02.06.2020).

\section{Informed Consent}

Informed consent was obtained from all individual participants included in the study.

\section{Consent for Publication}

Informed consent was obtained from all individual participants included in the study for publication of clinical research data.

\section{Availability of Data and Material}

Yes.

\section{Code Availability}

Yes.

\section{Author Contributions}

Pritish Singh, the Chief Operating Surgeon for most cases, wrote the manuscript and conducted data analysis. Dhananjay Sabat operated and assisted in surgeries and data analysis. Prashanth B assisted in surgeries and record keeping. Anubhav Vichitra assisted in surgeries, patient's follow-up and record keeping. Vinod Kumar assisted in clinical supervision of the patients and data analysis and edited the manuscript. All authors commented on previous versions of the manuscript. All authors read and approved the final manuscript.

\section{References}

1. Keshet $D$, Eidelman M. Clinical utility of the Taylor spatial frame for limb deformities. Orthop Res Rev 2017;9:51-61. DOI: 10.2147/ORR. S113420.

2. Hughes A, Parry M, Heidari N, et al. Computer hexapod-assisted orthopaedic surgery for the correction of tibial deformities. J Orthop Trauma 2016;30(7):e256-e261. DOI: 10.1097/BOT.0000000000000544.

3. Ganger R, Radler C, Speigner B, et al. Correction of post-traumatic lower limb deformities using the Taylor spatial frame. Int Orthop 2010;34(5):723-730. DOI: 10.1007/s00264-009-0839-5.

4. Manner HM, Huebl M, Radler C, et al. Accuracy of complex lowerlimb deformity correction with external fixation: a comparison of the Taylor Spatial Frame with the llizarov ring fixator. J Child Orthop 2007;1(1):55-61. DOI: 10.1007/s11832-006-0005-1.

5. Solomin LN, Paley D, Shchepkina EA, et al. A comparative study of the correction of femoral deformity between the llizarov apparatus and Ortho-SUV Frame. Int Orthop 2014;38(4):865-872. DOI: 10.1007/ s00264-013-2247-0.

6. Eren I, Eralp L, Kocaoglu M. Comparative clinical study on deformity correction accuracy of different external fixators. Int Orthop 2013;37(11):2247-2252. DOI: 10.1007/s00264-013-2116-x.

7. Solomin LN, Utekhin Al, Vilensky VA. Deformity correction and fracture treatment using the software-based ortho-SUV frame. In: The basic principles of external skeletal fixation using the ilizarov and other devices. Milan: Springer, 2012, pp. 705-803.

8. Wright J, Sabah SA, Patel S, et al. The silhouette technique: improving post-operative radiographs for planning of correction with a hexapod external fixator. Strat Traum Limb Recon 2017;12(2):127-131. DOI: 10.1007/s11751-017-0287-5.

9. Tetsworth KD, Paley D. Accuracy of correction of complex lowerextremity deformities by the Ilizarov method. Clin Orthop Relat Res 1994;301:102-110. Available at: https://pubmed.ncbi.nlm.nih. gov/8156660/

10. Paley D. Rotation and angulation-rotation deformities. In: Principles of deformity correction. Berlin: Springer, 2002, pp. 235-268.

11. Paley D. History and science behind the six-axis correction external fixation devices in orthopaedic surgery. Oper Tech Orthop 2011;21(2):125-128. DOI: 10.1053/j.oto.2011.01.011.

12. Vilensky VA, Pozdeev AP, Bukharev EV, et al. Orthopedic hexapods: history, present and prospects. Pediatr Traumatol Orthop Reconstr Surg 2015;3(1):61-69. DOI:10.17816/PTORS3161-69. 